\title{
ON THE STABILITY OF SMALL CLUSTERS \\ OR CLUSTER REMNANTS
}

\author{
L. O. LODÉN \\ Astronomical Observatory, Uppsala, Sweden \\ and \\ H. RICKMAN \\ Stockholm Observatory, Saltsjöbaden, Sweden
}

\begin{abstract}
There is strong evidence that the occurrence of mutually very nearby stars with identical spectral type and apparent magnitude is in many cases appreciably more frequent than one has reason to expect if there should only be a case of pure random combination. This phenomenon might be associated with the problem of stability of small stellar systems, if we can identify a certain number of the coincidences as some sort of cluster remnants. Besides the fact that there are several physical explanations of the coincidence phenomenon without consideration to dynamical stability conditions, there is also more than one stability aspect which may be regarded as reasonable. In the first instance we have to survey the possibilities that the stability of certain conglomerations of stars - particularly those with two components - may depend on the mass relation and will reach a maximum value for equal masses. As most straightforward we regard the conception that the most massive stars in a cluster (or even a multiple system) form the most stable and hence the most long-lived configuration, so that a cluster remnant most probably will consist of stars of rather equal mass.
\end{abstract}

\section{Introduction}

At visual inspection of objective-prism plates of Milky Way regions one frequently detects pairs - or occasionally higher multiples - of spectra of identical type and apparent magnitude and so close together on the plate that they form a conspicuous configuration (Lodén, 1969, 1973). In connection with a spectral survey of the southern Milky Way (Nordström and Sundman, 1973) about 2000 coincidence phenomena of this type with stars brighter than $m=14$ have been detected in the Carina-CruxCentaurus-Norma region $\left(l=280^{\circ}-330^{\circ}\right)$. The most common spectral type of the objects is $A(36 \%)$, followed by $B(31 \%)$ and $F(13 \%)$. For stars of type B and A brighter than $m=13$ the number of detected coincidences overwhelmingly exceeds the one expected in a random distribution. For fainter stars the excess becomes less pronounced and finally it turns into a deficit. This, however, is to a large extent connected with detection problems, and the real distribution of the coincidences for faint stars is difficult to estimate from the present material. The angular separation between the components ranges between 0.1 and $20^{\prime}$, and the correspondingly estimated linear separation in the transverse direction will range from 0.001 to 10 parsec.

\section{Possible Explanations of the Phenomenon}

There is no indication whatsoever that the coincidence phenomenon may be explained in terms of a unique astronomical feature. Probably there are a series of different circumstances, which in different cases cause two or more apparently equal stars to 
appear close to the same line of vision from the observer. The following ones may be suggested:

(a) A projection effect of two stars with a certain difference in luminosity and a rather large mutual distance along the line of sight. In this case the stars would have no directly physical association with each other.

(b) A close but temporary encounter between two equal stars of different origin.

(c) A binary (or multiple) system with components either of accidentally coinciding types, or with a physically conditioned identity (Stock, 1972).

(d) Accidentally coinciding members of a cluster or association which is not dense enough to contrast against the general stellar background.

(e) A cluster remnant consisting of two or more stars of approximately equal mass.

It should be emphasized that this list is probably not complete, and there is reason to believe that all the above explanations, and possibly a few more, are relevant in at least some of the cases. However, the different cases do certainly not occur with the same frequency. In our opinion, the explanations (d) and (e) should predominate over the other ones (Lodén, 1973).

\section{Explanations with Relevance to the Problems of Stability of Small Stellar Systems}

It seems that the indicated explanation (e) above will be the most interesting one to investigate with consideration to stability problems. This is due to the circumstance that if the explanation in question is preponderant, a tendency towards favouring of equal components in cluster remnants can be considered as observationally supported.

Next we have to look for a reasonable physical explanation of such a phenomenon. It is obvious then that no cluster with a restricted number of members can be absolutely stable. That is, external forces caused by, for instance, differential galactic rotation, passing stars and gas clouds and perhaps nearby clusters, and in addition mutal interaction between the members themselves will cause stars to leave the cluster. It has also been shown (e.g. Michie, 1963) that this 'evaporation' to a maximum extent influences the low-mass stars.

We may therefore have the situation that originally rich clusters are now highly impoverished and contain, say five to ten stars, all of which are of great mass. This in turn implies that the masses (and hence the spectral types) of these stars are approximately equal. If, on the other hand, we had a cluster which originally contained only a few stars of various masses, it seems more uncertain whether the remnant of such a cluster should consist of the two most massive members. It would certainly be of great interest to investigate to what extent one could expect that situation.

It seems reasonable, however, to interpret the majority of the coincidences as some kind of cluster remnants, whether the case (d) or the case (e) is relevant. Obviously the explanation (d) does really exist. Coincidences have in fact been found in established clusters. A special observation programme intended to give a clue to the problem about the importance of explanation (d) is at present in progress.

Explanation (d) is also interesting with consideration to stability problems, because 
whether or not the objects are remnants of rich clusters they indicate the presence of a great number of small clusters in space. This number appears at maximum if the coincidences are only accidental, and becomes reduced if the coincidences are conditioned by some physical phenomenon, for example that two massive stars or two stars of equal mass may tend to occur close together in a cluster (or elsewhere). Such an effect would directly lead to the situation suggested above, i.e. a favouring of equal components in cluster remnants, because two massive stars at short distance compared to the size of the cluster may form a subsystem of relatively great stability and is likely to be found in the remnant of the cluster. It is therefore an urgent mission to find out theoretically whether this effect is to be expected or not. Before we proceed to a more detailed discussion about this point, we mention a few previous results of interest.

A depletion of low-luminosity - or low-mass stars in open clusters compared to the solar neighbourhood luminosity function has been found, and this depletion can be explained as a result of the 'differential evaporation' of low-mass stars according to Michie (1963). Oort (1957) drew attention to the fact that open clusters older than about $5 \times 10^{8} \mathrm{yr}$, i.e. those with earliest main-sequence members of type A1 or later, are almost missing. Spitzer (1958) found that this time is consistent with the time-scale for disruption of an open cluster by collisions with interstellar clouds. It is interesting to note that the A-type coincidences are the most common ones, and they might thus reveal the remnants of the disrupted clusters.

\section{On the Existence and Persistence of Close Pairs of Massive Stars in Clusters}

We now consider a question which might be important for the understanding of the coincidence phenomena: What are the theoretical conditions for the formation of close pairs of stars of equal mass in a cluster and how permanent would such a configuration be? It should be realized that this general problem is extremely difficult, and it would probably be necessary'to study several cluster models with a computer and to analyze the results statistically in order to get a clear picture of the general situation. We will instead consider only some very simple concepts in a very simple model sketching the rough outlines.

To begin with, we consider two very massive stars instead of any two with equal mass. This is justified by the fact that the observed coincidences are formed by massive stars in a majority of the cases. Criticism may be raised against consideration of an isolated cluster since, in practice, there are no such ones, but on the other hand, if a certain result is found for an isolated cluster we may later on extend the discussion to concern a case of external influence.

Consider a cluster consisting of $N$ stars of mass $m$ and two stars of mass $m^{\prime}>m$. The only quantities for which we have some relation are the total energy and angular momentum plus the virial of the cluster. This can be expressend through the quantity $I$ given by:

$$
I=\frac{1}{N m} \sum_{i, j} m_{i} m_{j} r_{i j}^{2}
$$


In a steady-state cluster we should expect $I=$ constant (Chandrasekhar, 1942). If we separate $I$ into three terms corresponding to (a) pairs of low-mass stars, (b) pairs of one high-mass and one low-mass star, and (c) the two high-mass stars, we find that the last term will be very small compared to the other two in realistic cases. Therefore a rearrangement of the stars so that the distance between the two high-mass members suddenly decreases would not have much influence on the virial of the cluster, and the steady-state situation will not be violated by such a rearrangement.

The total potential energy $\Omega$, on the other hand, is more sensitive to changes in the contribution $\Omega^{\prime}$ by the two massive members. For a cluster with an 'average radius' $R$, where the two high-mass members are situated at mutual distance $r$, the ratio of the two energies is:

$$
\frac{\Omega^{\prime}}{\Omega} \sim \frac{2}{N^{2}}\left(\frac{m^{\prime}}{m}\right)^{2} \cdot\left(\frac{R}{r}\right) .
$$

If we assume $N=50, m^{\prime}=5 m, r=0.1 R$, we get $\Omega^{\prime}=0.2 \Omega$, i.e. a large fraction of the total potential energy of the cluster is stored in the mutual attraction of the two massive members. During the evolution of clusters it must frequently happen that two massive stars occur at such a short distance from each other, and the question is: What will happen then? Is their velocity of encounter so high that they will at once separate again? The answer is negative. If the encounter velocity is $v$ the condition for immediate breakup of the system gives a maximum distance $r_{\max }$ depending upon $v$, and if we use the velocity dispersion of the low-mass cluster members, corrected for the fact that heavier stars move at correspondingly lower velocities in a steady state, we arrive at the condition for 'internal' stability of the pair:

$$
r<r_{\max } \sim \frac{2 R}{N}\left(\frac{m^{\prime}}{m}\right)^{2} .
$$

With the assumptions above we find that $r \ll r_{\max }$. Therefore, if the other members of the cluster shall split up this pair by their gravitational action, a large binding energy - of the order of $\Omega$ - has to be overcome.

We expect that the most active 'splitting-up process' will be close encounters with other members of the cluster. Some of these will increase the total energy of the pair and others will decrease it, so that the effect will be a dispersion of the energy increasing with time. The question is, if this dispersion grows rapidly enough to split up the pair before the whole cluster has been disrupted. It seems that in a dense cluster with many members the process proceeds so rapidly that one should, in general, not expect any stable pairs of massive stars. On the other hand, in more dispersed clusters or poor ones in the stage of breaking up such close pairs of massive members should be able to persist for much longer times:

In conclusion to this section we may state that if we consider dispersed clusters or eventually cluster remnants containing a small number of stars, it seems that theoretically we can expect coincidences of massive stars to appear more frequently than 
would be the case in a pure random configuration. We have no quantitative information about this, however, and furthermore there remains to study the effect of interaction between the star couple and other stars of approximately equal mass and the influence upon the close pairs of massive stars during the disruption of the cluster due to external forces.

\section{Remarks About the Sketched Ideas}

As critical remarks about the principal ideas in this contribution we mention the following: Only two of the explanations above of the coincidence phenomenon give significance to the discussion performed. Suppose, for instance, that most of the stars with coinciding spectral type constitute some sort of twins or triplets etc. created by cleavage into equal parts of rapidly spinning stars. In such a situation most of the ideas would have no foundation in reality. Besides this fact, it is not at all certain that open clusters constitute a unique type of stellar systems from physical point of view, particularly as far as the dynamical constitution and the stability are concerned. Different clusters may well show quite different dynamical properties, internally as well as in relation to the surrounding field and neighbouring clusters. An explanation in terms of clusters or cluster remnants should therefore not imply an attempt to force the groupings of stars into a certain theoretical pattern deduced from simplified models assuming the existence of a well-defined center of mass, extremely simplified mass distribution, a Maxwellian velocity distribution or other formalistic regularities.

It should also be remarked that the stability is never uniquely mass-conditioned, and that we shall expect a few cluster remnants that contain no particularly massive stars. Furthermore, we must again draw attention to problems connected with the persistence of close pairs of stars under external influences and in the internal gravitational potential of a small system of stars having approximatively equal mass. Besides, we have as yet no explanation to the extreme similarity between many coincidence components, considering the fact that in a real cluster there are stars of, roughly speaking, any mass.

We emphasize, however, that the discussion above is directly inspired by observational experience and thereby put in connection with open clusters. On the other hand, if some results can be reached about general stability aspects or if some 'stability rules' may be deduced from further studies of these coincidences, it is quite conceivable that these results might also have implications for other kinds of systems - such as associations or clusters of galaxies.

\section{Prob̉ems Concerning Empirical Check}

No theoretical treatment of the stability problem can be considered as meaningful until it gets into contact with observational evidence. In the case of open clusters, the empirical check most near at hand would be performed by means of a statistical 
study of the distribution of spectral types in established clusters - particularly as a function of the number of stars in the cluster. One would then get an idea about the change of the mass distribution with decreasing number of members. As far as possible, this should also be combined with an age classification of the clusters. A convincing result - positive or negative - of such a study would give a rather decisive information about the applicability of the theory.

Unfortunately, it is very difficult to estimate the number of stars in a cluster, as the apparent one is mainly conditioned by limiting magnitude and density of the general stellar background. It is particularly troublesome to obtain spectra of a reasonable number of cluster members. Consequently there is a considerable risk for selection effects.

Furthermore, the detection completeness is highly dependent upon the density and dimensions of the cluster. Small and poor clusters are much more difficult to discover

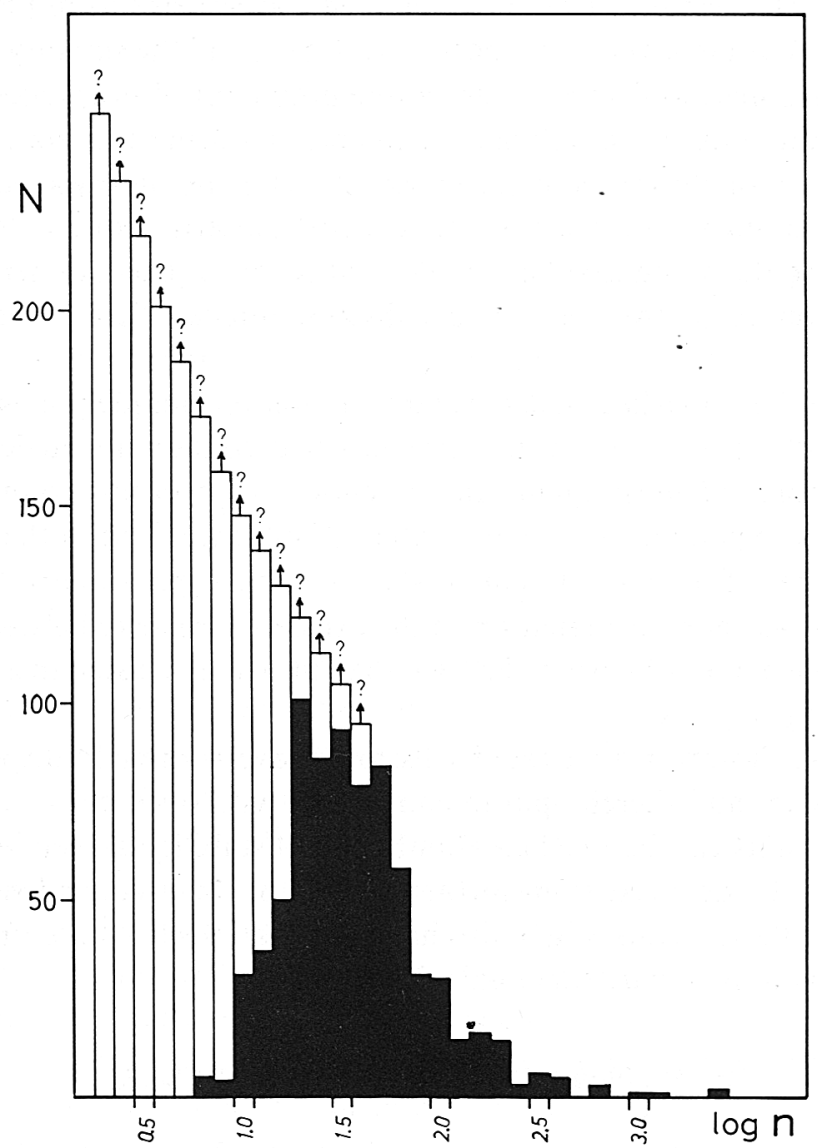

Fig. 1. Filled blocks show the apparent distribution of open clusters as a function of the logarithm of the indicated number of members. The open extensions indicate the suggested tentative extrapolation for the poor clusters which are difficult to detect. 
than the large rich ones. If we make an attempt to give the apparent frequency of open clusters as a function of the quoted number of members according to the information found in the catalogue by Alter et al. (1970) we obtain the result shown in Figure 1. According to that figure there should be a maximum of clusters with about 25 members and a rapid decline towards the poorer ones. In our opinion, however, this picture is misleading because of the detection function and possibly there should in reality be a continuous increase of the frequency towards the two-star clusters $(\log n=0.3)$ as tentatively indicated in the figure.

It is therefore obvious that we cannot immediately apply the suggested checking procedure without a preliminary estimation of the true distribution of clusters with respect to their physical parameters.

\section{Hints About Future Studies}

For a fruitful continuation of the studies of cluster stability it is necessary to proceed with both observational and theoretical work. As far as the observations are concerned, we find it most urgent to continue the search for small star-poor clusters in order to get a better knowledge about the correlation between the number of members and the frequency of clusters than the one of today. Then there should also be performed a scrutiny of the stellar content with respect to spectral type for a representative selection of clusters so that we may obtain a better estimate of the correlation star number - spectral type.

On the theoretical side it is important to proceed with attempts to solve the stability problem and determine the evolution with time of a variety of the relevant parameters, in the first instance mass distributions of the cluster members and the topographic composition of the cluster (correlation between mass and coordinates). The cluster models subject to stability studies up to now have been very simplified and idealized and one has a feeling that they may well have been oversimplified and overidealized.

\section{References}

Alter, G., Balázs, B., and Ruprecht, J.: 1970, Catalogue of Star Clusters and Associations,

Chandrasekhar, S.: 1942, Principles of Stellar Dynamics, University of Chicago Press, Chicago, p. 200.

Lodén, L. O.: 1969, Vistas in Astronomy 11, 161.

Lodén, L. O.: 1973, Astrophys. Space Sci. 24, 511.

Michie, R. W.: 1963, Monthly Notices Roy. Astron. Soc. 126, 331.

Nordström, B. and Sundman, A. : 1973, in C. Fehrenbach and B. E. Westerlund (eds.), 'Spectral Classification and Multicolour Photometry', IAU Symp. 50, 85.

Oort, J. H.: 1958, Pont. Acad. Sci. 16, 415.

Spitzer, L.: 1958, Astrophys. J. 127, 17.

Spitzer, L. and Härm, R.: 1958, Astrophys. J. 127, 544.

Stock, J. and Wroblewski, H.: 1972, Astron. Astrophys. 18, 341.

\section{DISCUSSION}

R. H. Miller: Do you consider your results to indicate the presence of a hierarchic composition of the Milky Way? 
L. O. Lodén: To some extent, yes.

$S$. R. Aarseth: According to my own calculations, the dynamical lifetime of a cluster will exceed that of stars earlier than A0. What is your comment to that?

L. O. Lodén: I admit that the situation is apparently critical for the B stars, but - on the other hand we might have underestimated the influence of external forces. 